\title{
Comparative metabolomics in vanilla pod and vanilla bean revealing the biosynthesis of vanillin during the curing process of vanilla
}

Fenglin $\mathrm{Gu}^{1,3^{*}}$, Yonggan Chen ${ }^{2}$, Yinghua Hong ${ }^{1}$, Yiming Fang ${ }^{1,3}$ and Lehe Tan ${ }^{1,3^{*}}$

\begin{abstract}
High-performance liquid chromatography-mass spectrometry (LC-MS) was used for comprehensive metabolomic fingerprinting of vanilla fruits prepared from the curing process. In this study, the metabolic changes of vanilla pods and vanilla beans were characterized using MS-based metabolomics to elucidate the biosynthesis of vanillin. The vanilla pods were significantly different from vanilla beans. Seven pathways of vanillin biosynthesis were constructed, namely, glucovanillin, glucose, cresol, capsaicin, vanillyl alcohol, tyrosine, and phenylalanine pathways. Investigations demonstrated that glucose, cresol, capsaicin, and vanillyl alcohol pathway were detected in a wide range of distribution in microbial metabolism. Thus, microorganisms might have participated in vanillin biosynthesis during vanilla curing. Furthermore, the ion strength of glucovanillin was stable, which indicated that glucovanillin only participated in the vanillin biosynthesis during the curing of vanilla.
\end{abstract}

Keywords: Vanilla curing, LC-MS, Metabolomics, Vanillin biosynthesis

\section{Introduction}

Vanilla is a tropical orchid, which originated in Mexico (Lubinsky et al. 2008). It is one of the most important and popular aromatic compound used in food, beverages, and cosmetics (Kaur and Chakraborty 2013; Korthou and Verpoorte 2007). The fruit of a fully grown and mature vanilla is called vanilla pod. Vanilla pods are flavorless, but develop a characteristic aroma during the curing process. Traditional curing process generally comprises four steps, namely killing, sweating, drying, and conditioning. Vanilla bean refers to the vanilla pod after the curing process (Frenkel et al. 2011; Mariezcurrena et al. 2008; Sreedhar et al. 2009).

Natural vanilla flavor comprises a large number of aromatic compounds, including the principal flavor component vanillin and over 200 other volatile compounds with delicate sweet fragrances (Sharp et al. 2012). Vanillin content varies with different curing processes used around

\footnotetext{
*Correspondence: xiaogu4117@163.com; Lehe_Tan@163.com

1 Spice and Beverage Research Institute, CATAS, Wanning 571533, Hainan, China

Full list of author information is available at the end of the article
}

the world. Madagascar produces the best quality vanilla, with a vanillin content of $2.0-3.4 \%$, whereas the Indian vanilla contains $1.0-2.0 \%$ vanillin (Ranadive 1994; Westcott et al. 1994; Röling et al. 2001). Despite the fact that vanillin is the most popular flavor and probably the most prevalent natural plant product, it is also an extremely simple molecule. Since the vanillin biosynthesis pathway remains unclear, various attempts to modernize and improve the curing process have been conducted, involving oven drying, solar drying, and enzyme treatment (Dignum et al. 2001). However, the biosynthesis of vanillin is not efficient, nor could the quality of the vanilla bean be easy to control.

Metabolomics, a powerful approach in investigating the characteristics of the low- molecular-weight metabolite present in a biological sample, has become an important part of systems biology, complementing genomics and proteomics (Sasaki et al. 2016). The main analytical techniques applied in metabolomics analysis are nuclear magnetic resonance (NMR) spectroscopy, gas chromatography-mass spectrometry (GC-MS), and liquid chromatography-mass spectrometry (LC-MS). These techniques facilitate analysis of a wide range of 
metabolites with diverse physicochemical properties occurring at different concentration levels (Dunn and Ellis 2005). However, GC-MS may be prone to confounding factors introduced by the sample derivatization process or thermal degradation of metabolites at elevated temperatures (Xu et al. 2010). Furthermore, NMR may be biased toward the detection of large abundance metabolites and is the least sensitive of the three techniques. LC-MS provides high sensitivity and be capable to detect a wide range of metabolites (Chen et al. 2011). Nevertheless, it is susceptible to retention time (RT) drift and matrix effects related to electrospray ionization (ESI). The downstream data processing is time-consuming and complex. Advanced data processing software algorithms and multivariate chemometric tools are needed to process and interpret data obtained in LC-MS-based metabolomics.

A previous study has shown that the colonizing Bacillus isolates produce $\beta$-D-glucosidase, which mediates glucovanillin hydrolysis and influences flavor formation (Chen et al. 2015). The study further indicated that the curing process would result in metabolite variation. However, only few reports on the effects of curing on the metabolite variations of vanilla have been conducted (Frenkel et al. 2011; Gallage et al. 2014; Dignum et al. 2002; Palama et al. 2010, 2012). In this study, we used liquid chromatography-mass spectrometry (LC-MS) and multivariate chemometric methods to characterize the metabolic changes between vanilla pod and vanilla bean, identifying characteristic metabolites, and interpreted these changes in order to reveal the biosynthesis pathway of vanillin.

\section{Materials and methods}

\section{Plant materials and chemicals}

Vanilla pods were collected in Hainan, China, and cured by hot air processing (Dong et al. 2014). HPLC grade methanol was purchased from Fisher Scientific (Fair Lawn, NJ, USA), and HPLC-grade acetonitrile and reagent grade formic acid were obtained from Merck KGaA (Darmstadt, Germany). Distilled water was purified with Milli-Q Integral System Millipore (Bedford, MA, USA).

\section{Sample preparation}

Thirty vanilla pods and cured vanilla beans were collected for LC-MS analysis. The sample was crushed and placed in a 96-well plate, then precipitated using acetonitrile/ methanol (50:50, v/v) solvent. After vacuum pump, each well was washed with $180 \mu \mathrm{L}$ of the precipitate rinsing solvent. After drying the sample, $200 \mu \mathrm{L}$ mixtures were used for extracting small molecular compounds, and the mixture was composed of 5\% acetonitrile solution/ methanol $(70: 30, v / v)$. Finally, the mixture was used for LC-MS analysis.

\section{Non-targeted metabolic profiling spectral acquisition}

The LC-MS system was run in gradient mode. Solvent A was $5 \%(\mathrm{v} / \mathrm{v})$ formic acid/mixture (10\% methanol/90\% water), and solvent $\mathrm{B}$ was acetonitrile. The flow rate was $0.2 \mathrm{~mL} / \mathrm{min}$. The total injection volume for analysis was $10 \mu \mathrm{L}$. A C-18 column $(150 \mathrm{~mm} \times 2.1 \mathrm{~mm}, 5 \mu \mathrm{m}$, Waters, USA) was used for all analyses. The gradient was as follows: $0-2 \mathrm{~min}, 5 \% \mathrm{~B} ; 2-3 \mathrm{~min}, 7 \% \mathrm{~B} ; 3-4 \mathrm{~min}, 8 \%$ B; 4-5 min, 70\% B; 5-9 min, 90\% B; 9-11 min, 95\% B; 11-14 $\mathrm{min}, 95 \%$ - 5\% B; and 14-15 min, 5\% B.

LC-MS data was acquired using high performanceliquid chromatography (HPLC) system (LC-20AD, Shimadzu Co., Kyoto, Japan) coupled online to a LTQ Orbitrap Velos instrument (Thermo LTQ-Orbitrap Velos, Bremen, Germany) set at 30,000 resolution. Sample analysis was carried out in positive ion modes. The mass scanning range was $50-1000 \mathrm{~m} / \mathrm{z}$, the total retention time was $1200 \mathrm{~s}$, and the capillary temperature was $350{ }^{\circ} \mathrm{C}$. Nitrogen sheath gas was set at a flow rate of $30 \mathrm{~L} /$ min. Nitrogen auxiliary gas was set at a flow rate of $10 \mathrm{~L} /$ min and the spray voltage was set to $4.5 \mathrm{kV}$.

\section{Data analysis}

For the extraction of LC-MS raw data, Xcalibur native acquisition was performed with XCMS. All data were processed by local XCMS with the following parameters: signal $/$ noise threshold $=3, \mathrm{ppm}=10$, peak width $=(20$, $50)$, and prefilter $=(3,1000)$. Retention time correction was performed with the standard obiwarp algorithm in XCMS with prfostep $=1$. To correct the MS response shift during the run, the raw data were normalized against total integration values. Principal component analysis (PCA), orthogonal partial least squares discriminant analysis (OPLS-DA), and hierarchical cluster analysis (HCA) were performed by R-2.14.2. The VIP value and the significance were expressed by using Student's $t$ test of R-2.14.2. VIP $>1$ and $p<0.05$ were considered significant. Pathway Builder Tool 2.0 was used to build the vanillin metabolic pathways.

\section{Metabolite identification}

KEGG.gff, HMDB.gff, HMDB-SERUM.gff, LIPID.gff, and MetaCyc.gff metabolism database were used to identify metabolites in the LC-MS chromatograms. For LC-MS data, the putative identities of each ion were first given within XCMS by matching features in the databases with the following parameters: $\mathrm{ppm}=20$, adducts $=[\mathrm{M}+\mathrm{H}]^{+}$, $\left[\mathrm{M}+\mathrm{NH}_{4}\right]^{+},[\mathrm{M}+\mathrm{Na}]^{+}$, and $[\mathrm{M}+\mathrm{K}]^{+}$in the positive ion mode. Furthermore, the potential molecule formulas of each ion were extracted from a database based on 
accurate mass. The metabolites were identified based on accurate mass, fragmentation pattern, and retention time.

\section{Results}

\section{Metabolic profiles of vanilla}

The extracts of vanilla samples were analyzed via the LC-MS technique, allowing the analyses of a wide range of untargeted compounds. The representative LC-MS total ion current (TIC) chromatographs of the extracts obtained from vanilla pods and vanilla beans are shown in Fig. 1. The abscissa and ordinate represent the retention time and $\mathrm{m} / \mathrm{z}$ value, respectively. Each point in the graph represents an ion. The color of each point corresponds to the ion signal intensity, following black $>$ blue $>$ green $>$ red $>$ white, with the ion signal intensity gradually increasing. The main $\mathrm{m} / \mathrm{z}$ values were in the range of 50-800, and the retention time was concentrated at 60-900 s.

Metabolic variations between vanilla pod and vanilla bean For each $\mathrm{m} / \mathrm{z}$ value, there is a LOESS value in the comparison group, and the average LOESS value of each sample was obtained. The ratio of LOESS value was taken for each $\mathrm{m} / \mathrm{z}$ corresponding to the change fold (differential multiple), and the change fold greater than 1.5 or less than 0.67 of the $\mathrm{m} / \mathrm{z}$ were selected. The logarithm of these change folds was used as the horizontal coordinate, and $Q$ value as the vertical coordinate mapping. Red dots represent significantly different $\mathrm{m} / \mathrm{z}$, for 1723 , accounting for $30.51 \%$; the blue points are significantly similar $\mathrm{m} / \mathrm{z}$, for 635 , accounting for $11.24 \%$; and gray points represent non-significant $\mathrm{m} / \mathrm{z}$, for 3290 , accounting for $58.25 \%$ (Fig. 2).

PCA can extract chemical information objectively, which has been well established for discrimination and grouping in analyzing metabolic profiles. PCA developed a visual plot for the evaluation of similarities and differences between the metabolic profiles of vanilla pods and vanilla beans on the basis of metabolites. Based on a correction of $0-30 \%$ RSD (QC sample not included), the LOESS value was used for PCA analysis (Fig. 3). Principal component 1 (PC1) accounted for the major differences in variances, whereas the principal component 2 (PC2) accounted for the minor differences. Fifteen vanilla pods were clustered together, which indicated that the metabolites were generally similar. On the contrary, vanilla beans were scattered, implying that the metabolites were quite different. Furthermore, vanilla beans showed significant differences from vanilla pods. The results showed that vanilla beans were greatly distinct even though they were cured from the same vanilla pods.

The metabolites exceeding in the PLS-DA (partial least squares-discriminant analysis) model and with

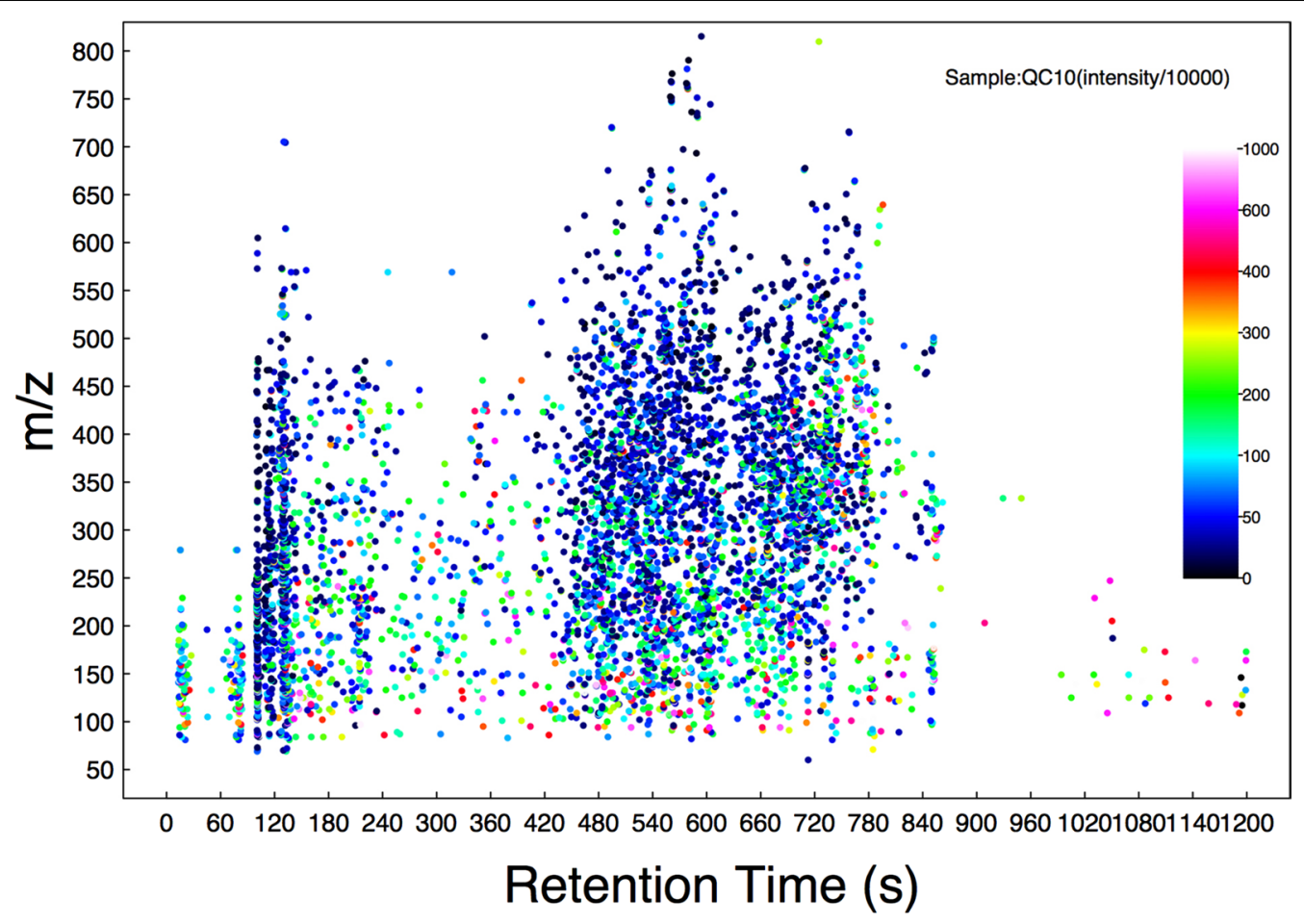

Fig. 1 A chromatogram of total ions 


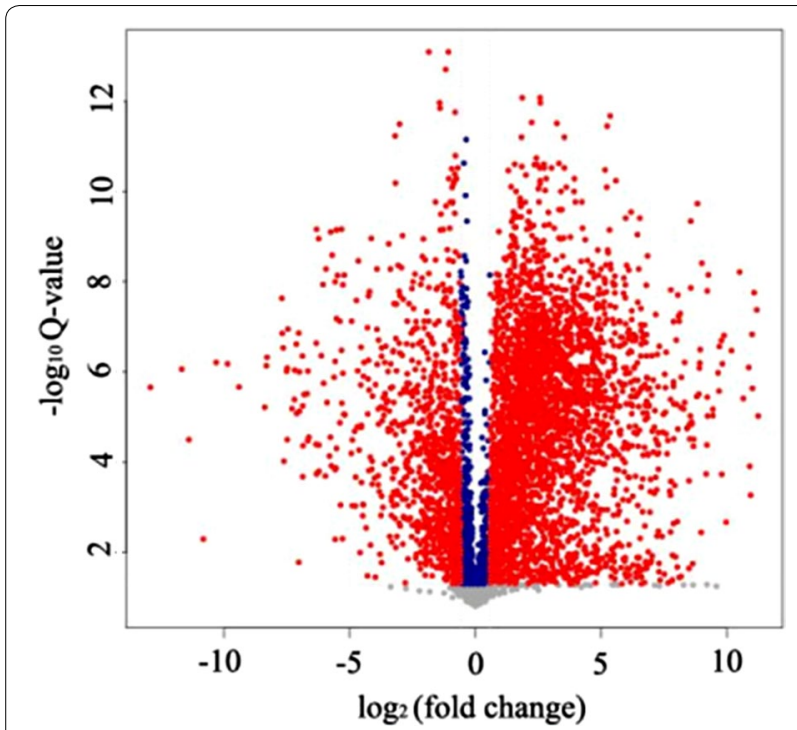

Fig. 2 Volcano plot between vanilla pods and vanilla beans [variables in red are significant $(Q<0.05)$, and variables in blue are not significant] a correction of $0-30 \%$ RSD data were selected to focus on the important marker metabolites distinguishing between vanilla pods and vanilla beans. The $\mathrm{m} / \mathrm{z}$ group corresponding to the LOESS value was compared using OSC (orthogonal signal correction) method to remove part of the noise, and then carried out by PLS-DA analysis. The results of the PLS-DA analyses were similar as PCA analysis. Vanilla beans considerably differed from each other, and they were also different from vanilla pods (Fig. 4).

A heat map was constructed based on the detection of all samples, with a total of $5648 \mathrm{~m} / \mathrm{z}$ values (Fig. 5). The results showed that the same group samples were quite similar, indicating the significant differences of metabolites in the fermentation of vanilla pods and vanilla beans.

\section{The speculated biosynthesis pathway of vanillin}

According to the results of the comparison of the $\mathrm{m} / \mathrm{z}$ values with the database, 6822 kinds of compounds were identified where 21 compounds related to vanillin synthesis were identified based on the reported biosynthesis

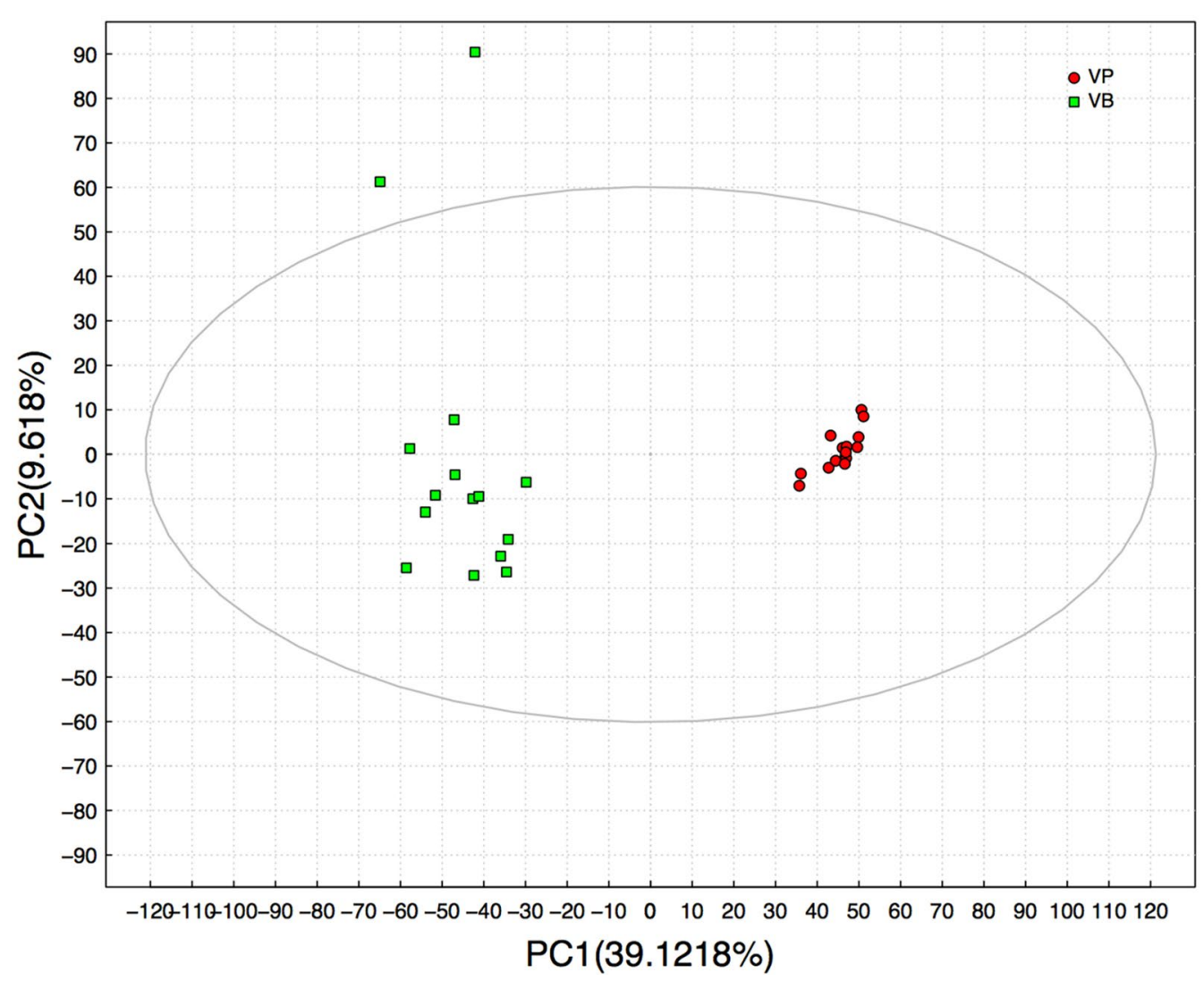

Fig. 3 PCA score separating vanilla pods and vanilla beans away from each other (VP vanilla pods, VB vanilla beans) 


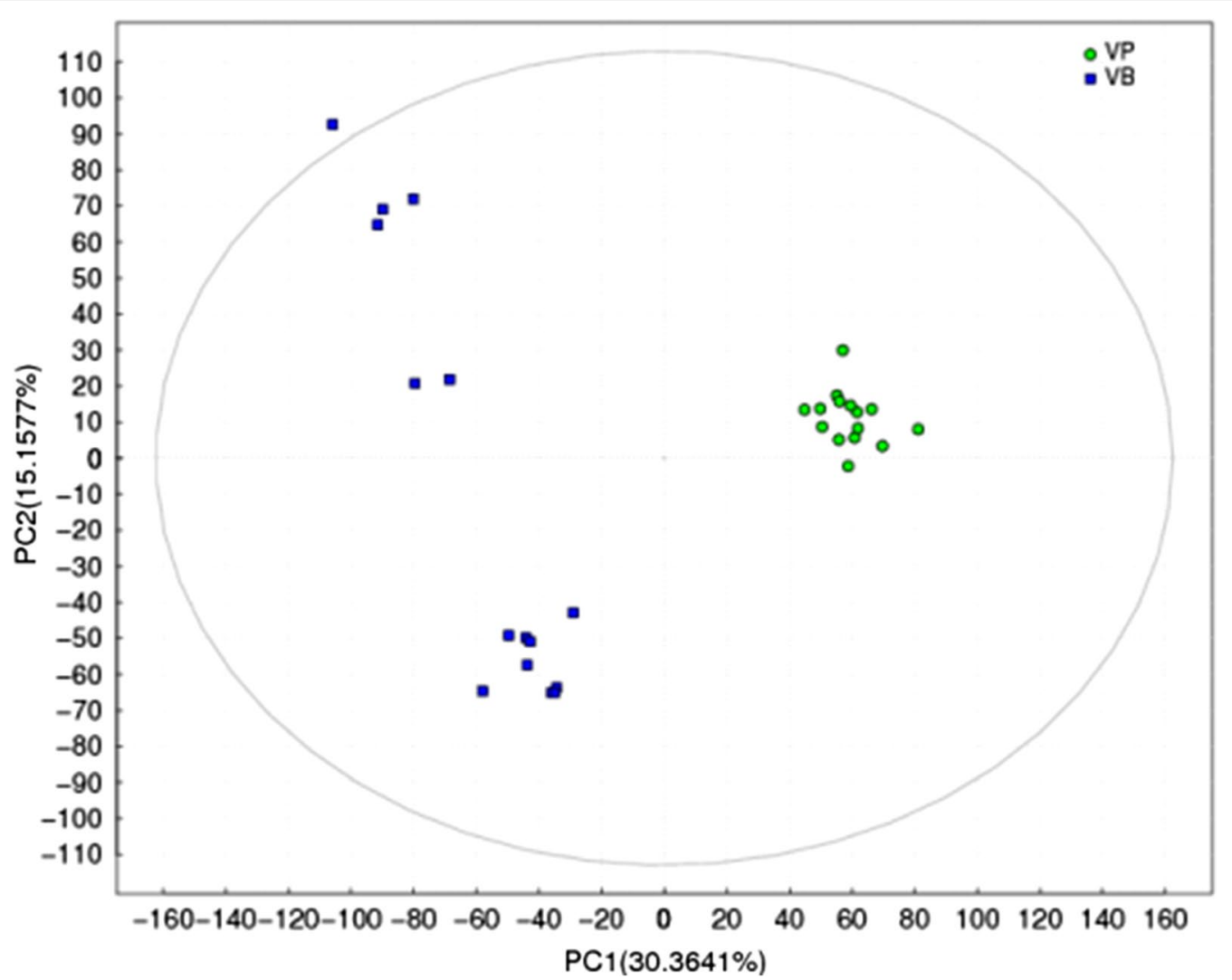

Fig. 4 PLS-DA score separating vanilla pods and vanilla beans away from each other (VP vanilla pods, VB vanilla beans)

pathway of vanillin. The ion strength of individual components changed and was related to vanillin biosynthesis as shown in Additional file 1: Figure S1. The ion strength of glucovanillin was stable in vanilla pods and vanilla beans, whereas the ion strength of most other compounds exhibited divergence.

The biosynthesis pathway of vanillin during fermentation was constructed based on the precursors of vanilla synthesis, as shown in Fig. 6. Previous studies reported that vanillin originated from $\beta$-D-glucosidase hydrolysis of glucovanillin (Dignum et al. 2004) or ferulic acid decarboxylation (Gallage et al. 2014). In our study, these two mentioned pathways were also found. In addition, the precursors of ferulic acid were also detected, namely L-phenylalanine and tyrosine. Moreover, this study also found four more vanillin synthesis pathways, which, in our knowledge, were not reported to occur in vanilla curing. These pathways distributed in a wide range of microbial metabolism (Kaur and Chakraborty 2013). The four newly-found pathways of vanillin biosynthesis were as follows: shikimic acid pathway of glucose by de novo synthesis; and oxidation of cresol, capsaicin, and vanilla alcohol.

\section{Discussion}

The curing process is an essential procedure for the production of vanilla flavor. This process induces destruction of tissue organization and reduces vanilla pod moisture. Furthermore, it creates conditions which allow the free flow of compartmentalized cellular constituents, resulting in enzyme-substrate interactions (Frenkel et al. 2011). Various biochemical and chemical reactions, such as esterification, etherification, and oxidative degradation, take place during this period to produce volatile compounds (Uzio and Derbesy 1989). However, the variations, especially those relating to the metabolism of vanilla fruits, still remain poorly constrained (Palama et al. 2009, 2011). Previous studies mainly focused on volatile compounds of vanilla beans but not on the overall metabolic fingerprints of vanilla fruits (Zhang and Christoph 2012; Pérez-Silva et al. 2006). This study applied untargeted metabolomic and chemometric analysis, indicating a notable differentiation between vanilla pods and vanilla beans. As shown in Figs. 3 and 4, PCA and PLS-DA demonstrated a clear and statistically significant separation of vanilla pods and vanilla beans. The substantial changes were observed in the metabolite composition 


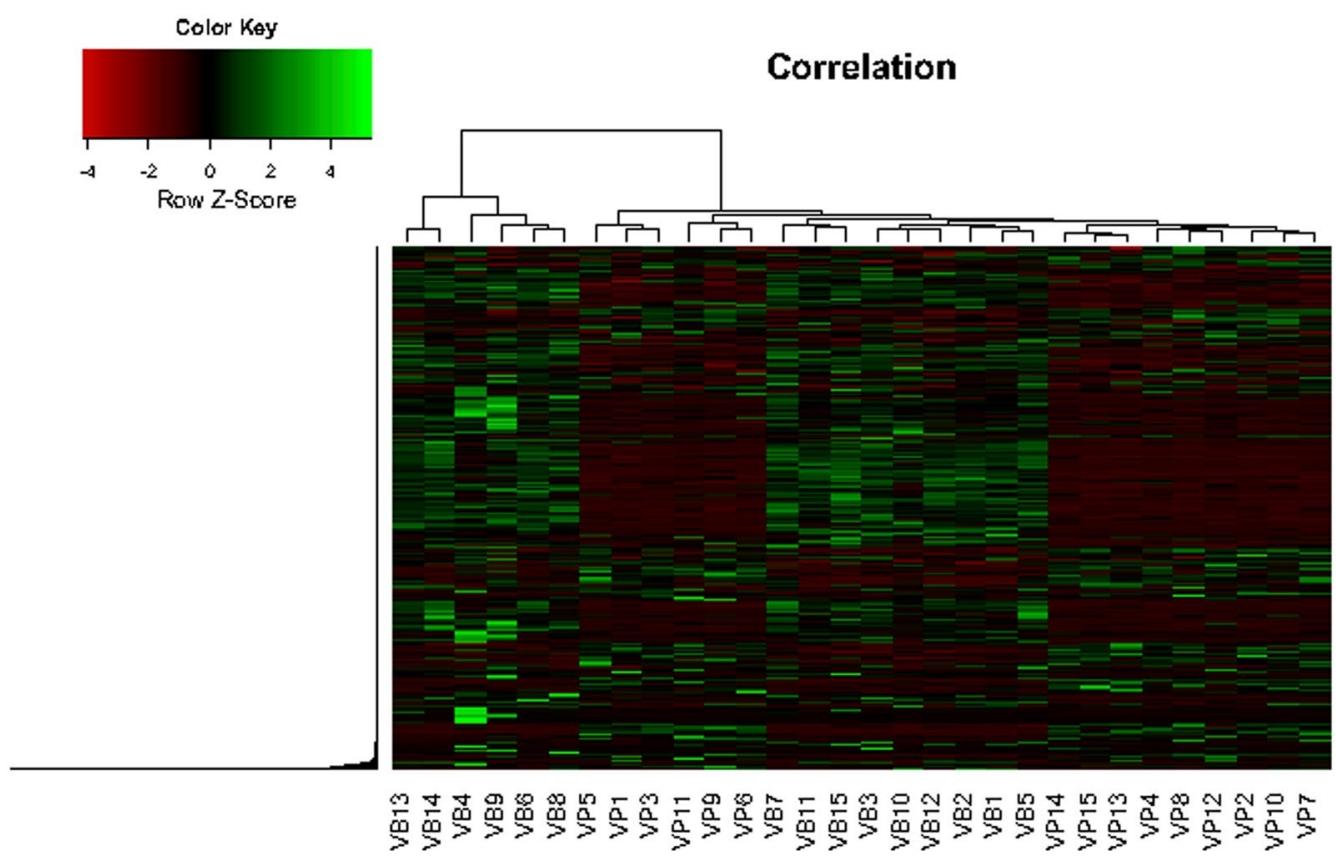

Fig. 5 Heat map of cluster for vanilla pods and vanilla beans

of vanilla beans. Results showed that the curing process could give rise to metabolite changes in vanilla beans even though they contained similar metabolites in vanilla pods. This could be attributed to the effect of the different environmental microbes during the curing process. As shown in Additional file 1: Figure S1, the stability of glucovanillin ion strength showed that it was mainly involved in the metabolic pathway of vanillin biosynthesis and did not participate in other metabolic ways during the curing process, and the ion strength divergence of the other compounds indicated they not only participated in the vanillin biosynthesis, but were also involved in other flavors formation by the microbial metabolism.

Biosynthesis of vanillin is of interest to researchers for two main reasons: the relationship of vanillin with the mechanisms of the formation of benzoic acids and with the phenylpropanoid pathway; and the commercial importance of vanillin and the possibilities of producing the compound by biotechnological routes (Kaur and Chakraborty 2013). Vanillin in green pods is present exclusively in conjugated form, principally as the glucovanillin, and the beans display no trace of the characteristic vanilla flavor at this stage (Odoux 2006; Walton et al. 2003). Vanillin only develops during the curing process. One of the most obvious aspects of curing is that glucovanillin reacts with $\beta$-D-glucosidase to form vanillin (Kanisawa et al. 1994; Ramachandra and Ravishankar 2000; Dignum et al. 2001). In addition, Zenk (1965) reported the results of radioactive label ferulic and vanillic acids, and proposed a route by which vanillin was derived from ferulic acid. A quite different route was later proposed based on enzyme assay (Reference needed, I guess it's Dixon 2011?). According to this route, vanillin was formed from coumaric acid by non-oxidative chain shortening or via any one of three Coenzyme A esters by $\beta$-oxidation. However, to our knowledge, this is the first time that the glucose, cresol, capsaicin, and vanillyl alcohol pathways were proposed, which implied that vanillin biosynthesis was not as simple as it seemed. Furthermore, the pathways and mechanisms elucidated in this study could aid future studies on the production of vanillin.

Various metabolic pathways of vanillin biosynthesis were found in bacteria, fungi, plant cells, and genetically engineered microorganisms. These metabolisms included the production of vanillin from lignin, eugenol, isoeugenol, phenolic stilbenes, ferulic acid, and amino acids (Zhao et al. 2005). However, only $p$-coumaric acid and ferulic acid were found as precursors in the vanilla and in vanilla cell cultures, which were involved in the shikimate and phenylalanine pathways (Gallage et al. 2014). In this study, three other biosynthesis pathways were evidenced: glucose, cresol and capsaicin oxidation. A flavoprotein vanillyl alcohol oxidase (vaoA) was essential for creosol and capsaicin to produce vanillin (Kaur and Chakraborty 2013). VaoA was found in a wide range of microorganisms, such as Dictyostelium discoideum, Cavia porcellus, Saccharomyces cerevisiae, Candida albicans, and 


\section{Vanillin Pathway}

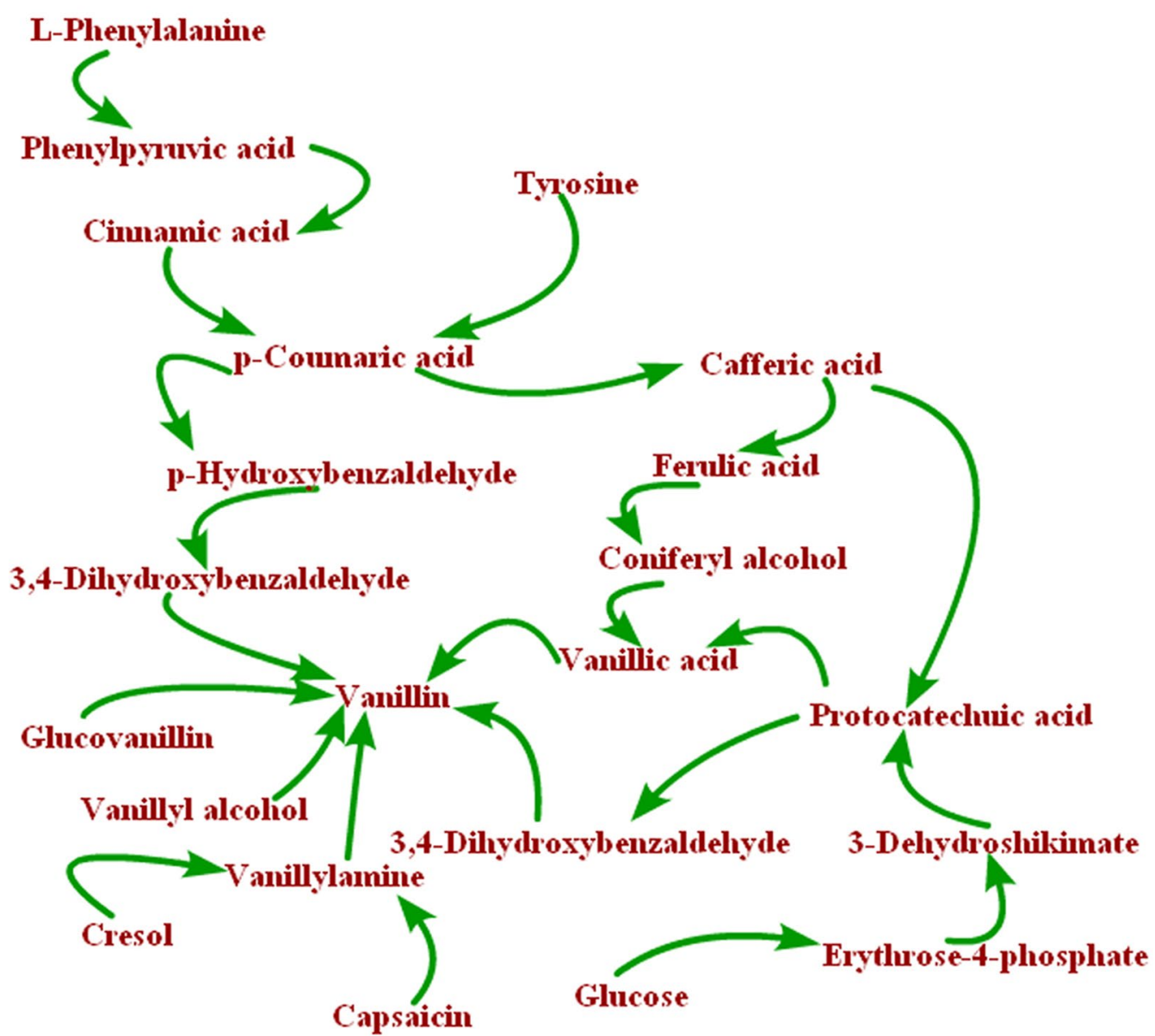

Fig. 6 Some presumed metabolic pathways of vanillin biosynthesis (cited from: the pathway of glucose, capsaicin, cresol, vanillyl alcohol, L-phenylalanine, tyrosine cited from Kaur and Chakraborty 2013; and the pathway of glucovanillin cited from Dignum et al. 2004)

Streptococcus pneumoniae (Leferink et al. 2008). Similarly, 3-dehydroshikimate dehydratase was essential in the glucose pathway via de novo biosynthesis, which was found in Emericella nidulans, Neurospora crassa, Klebsiella pneumoniae, Vigna mungo, Bacillus thuringiensis, and Podospora pauciseta (Lamb et al. 1992; Draths and Frost 1995; Fox et al. 2008; Hansen et al. 2009). However, only few reports were known regarding the presence of two enzymes in Orchidaceae (Hansen et al. 2009).

The metabolic profiles of vanilla pods and vanilla beans illustrated that the metabolome of vanilla fruits were mainly dominated by $5648 \mathrm{~m} / \mathrm{z}$. According to the $\mathrm{m} / \mathrm{z}$ analysis, the vanilla pods showed significant differences from vanilla beans. In addition, 21 precursors related to vanillin synthesis were identified, and 7 pathways of vanillin biosynthesis were constructed, including glucovanillin, glucose, cresol, capsaicin, vanillyl alcohol, tyrosine, and phenylalanine pathways. To the best of our knowledge, the glucose, cresol, capsaicin, and vanillyl alcohol pathways have not yet been reported in any vanilla curing process. However, these pathways have wide range of distribution in microbial metabolism. Therefore, microorganisms may have participated in vanillin biosynthesis during the curing of vanilla.

\section{Additional file}

Additional file 1: Figure S1. A part of components change in the process and relate to the vanillin biosynthesis. a. Erythrose-4-phosphate; b. Glucose; c. p-Hydroxybenzaldehyde; d. 3,4-Dihydroxybenzaldehyde; e. Protocatechuic acid; f. Cresol; g. 3-Dehydroshikimate; h. Vanillin; i. Vanillic acid; j. Vanillyl alcohol; k. Vanillylamine; I. Cinnamic acid; m. Phenylpyruvic acid, p-Coumaric acid; n. Cafferic acid; o. L-Phenylalanine; p. Tyrosine; q. Ferulic acid; r. Coniferyl alcohol; s. Glucovanillin; t. Capsaicin. 


\section{Abbreviations}

LC-MS: high-performance liquid chromatography-mass spectrometry; NMR: nuclear magnetic resonance; GC-MS: gas chromatography-mass spectrometry; RT: retention time; ESI: electrospray ionization; HPLC: high performanceliquid chromatography; PCA: principal component analysis; OPLS-DA: orthogonal partial least squares discriminant analysis; HCA: hierarchical cluster analysis; TIC: total ion current; PLS-DA: partial least squares-discriminant analysis; OSC: orthogonal signal correction; vaoA: flavoprotein vanillyl alcohol oxidase.

\section{Authors' contributions}

FLG collected the samples, performed the experiments, analyzed the data, and wrote the paper. YGC and LHT helped in performing the experiments and analyzed the data. YHH and YMF critically analyzed the study and helped in drafting the article as well as edited the manuscript. All authors read and approved the final manuscript.

\section{Author details}

${ }^{1}$ Spice and Beverage Research Institute, CATAS, Wanning 571533, Hainan, China. ${ }^{2}$ College of Tropical Biology and Agronomy, Hainan Tropical Ocean University, Sanya 572022, Hainan, China. ${ }^{3}$ Key Laboratory of Genetic Resources Utilization of Spice and Beverage Crops, Ministry of Agriculture, Wanning 571533, Hainan, China.

\section{Acknowledgements}

The authors are thankful to National Science Foundation of China and Ministry of Science and Technology for the funding.

\section{Competing interests}

The authors declare that they have no competing interests.

\section{Availability of data and materials}

The datasets supporting the conclusions of this article are included within the article and its Additional file 1.

\section{Consent for publication}

This article does not contain individual person's data.

\section{Ethics approval}

This article does not contain any studies with human participants or animals performed by any of the authors.

\section{Funding}

This study was funded by the National Science Foundation of China (31671848) and the National Science and Technology Support Program (2012BAD36B03).

\section{Publisher's Note}

Springer Nature remains neutral with regard to jurisdictional claims in published maps and institutional affiliations.

Received: 13 April 2017 Accepted: 26 May 2017

Published online: 05 June 2017

\section{References}

Chen LY, Zhou L, Chan ECY, Neo J, Beuerman RW (2011) Characterization of the human tear metabolome by LC-MS/MS. J Proteome Res 10:4876-4882

Chen YG, Gu FL, Li JH, He SZ, Xu F, Fang YM (2015) Involvement of colonizing Bacillus isolates in glucovanillin hydrolysis during the curing of Vanilla planifolia Andrews. Appl Environl Microb 81:4947-4954

Dignum MJW, Kerler J, Verpoorte R (2001) Vanilla production: technological, chemical, and biosynthetic aspects. Food Rev Int 17:199-219

Dignum MJW, Kerler J, Verpoorte R (2002) Vanilla curing under laboratory conditions. Food Chem 79:165-171

Dignum MJW, Heijden RVD, Kerler J, Winkel C, Verpoorte R (2004) Identification of glucosides in green beans of Vanilla planifolia Andrews and kinetics of vanilla $\beta$-glucosidase. Food Chem 85:199-205
Dixon RA (2011) Curing of vanilla. In: Havkin-Frenkel D, Belanger FC (eds) Handbook of vanilla science and technology, vol 1. Wiley-Blackwell, Oxford, pp 292-298

Dong ZZ, Gu FL, Xu F, Wang QH (2014) Comparison of four kinds of extraction techniques and kinetics of microwave-assisted extraction of vanillin from Vanilla planifolia Andrews. Food Chem 149:54-61

Draths K, Frost J (1995) Environmentally compatible synthesis of catechol from D-glucose. J Am Chem Soc 117:2395-2400

Dunn WB, Ellis DI (2005) Metabolomics: current analytical platforms and methodologies. Trac Trend Anal Chem 24:285-294

Fox DT, Hotta K, Kim CY, Koppisch AT (2008) The missing link in petrobactin biosynthesis: asbF encodes a (-)-3-dehydroshikimate dehydratase. Biochemistry 47:12251-12253

Frenkel C, Ranadive AS, Vázquez JT, Havkin-Frenkel D (2011) Curing of vanilla. In: Havkin-Frenkel D, Belanger FC (eds) Handbook of vanilla science and technology, vol 1. Wiley-Blackwell, Oxford, pp 84-96

Gallage NJ, Hansen EH, Kannangara R, Olsen CE, Motawia MS, Jøgensen K, Holme I, Hebelstrup K, Grisoni M, Møllera BL (2014) Vanillin formation from ferulic acid in Vanilla planifolia is catalysed by a single enzyme. Nat Commun 5:1-14

Hansen EH, Moeller BL, Kock GR, Bunner CM, Kristensen C, Jensen OR, Okkels FT, Olsen CE, Motawia MS, Hansen J (2009) De novo biosynthesis of vanillin in fission yeast (Schizosaccharomyces pombe) and baker's yeast (Saccharomyces cerevisiae). Appl Environ Microb 75:2765-2774

Kanisawa T, Tohoro K, Kawakara S (1994) Flavor development in the beans of Vanilla planifolia. In: Kurihara K, Suzuki N, Ogawa H (ed) Olfaction taste. Proceedings of the 11th international symposium. Springer, Tokyo, pp 268-270

Kaur B, Chakraborty D (2013) Biotechnological and molecular approaches for vanillin production: a review. Appl Biochem Biotech 169:1353-1372

Korthou H, Verpoorte R (2007) Vanilla. In: Berger RG (ed) Flavours and fragrances, vol 1. Springer, Berlin, pp 203-217

Lamb HK, Van den Hombergh JPTW, Newton GH, Moore JD, Roberts CF, Hawkins AR (1992) Differential flux through the quinate and shikimate pathways. Implications for the channeling hypothesis. Biochem J 284:181-187

Leferink NGH, Heuts DPHM, Fraaije MW, Berkel WJHV (2008) The growing VAO flavoprotein family. Arch Biochem Biophys 474:292-301

Lubinsky P, Bory S, Hernandez JH, Kim SC, Gomez-Pompa A (2008) Origins and dispersal of cultivated vanilla (Vanilla planifolia Jacks. [Orchidaceae]). Econ Bot 62:127-138

Mariezcurrena MD, Zavaleta HA, Waliszewski KN, Sánchez V (2008) The effect of killing conditions on the structural changes in vanilla (Vanilla planifolia, Andrews) pods during the curing process. Int J Food Sci Tech 43:1452-1457

Odoux E (2006) Glucosylated aroma precursors and glucosidase(s) in vanilla bean (Vanilla planifolia G. Jackson). Fruits 61:171-184

Palama TL, Khatib A, Choi YH, Payet B, Fock I, Verpoorte R, Kodja H (2009) Metabolic changes in different developmental stages of Vanilla planifolia pods. J Agric Food Chem 57:7651-7658

Palama TL, Fock I, Choi YH, Verpoorte R, Kodja H (2010) Biological variation of Vanilla planifolia leaf metabolome. Phytochemistry 71:567-573

Palama TL, Khatib A, Choi YH, Côme B, Fock I, Verpoorte R, Kodja H (2011) Metabolic characterization of green pods from Vanilla planifolia accessions grown in La Réunion. Environ Exp Bot 72:258-265

Palama TL, Grisoni M, Fock-Bastide I, Jade K, Bartet L, Choi YH, Verpoorte R, Kodja H (2012) Metabolome of Vanilla planifolia (Orchidaceae) and related species under Cymbidium mosaic virus (CymMV) infection. Plant Physiol Biochem 60:25-34

Pérez-Silva A, Odoux E, Brat P, Ribeyre F, Rodriguez-Jimenes G, Robles-Olvera V, García-Alvaradob MA, Günata Z (2006) GC-MS and GC-olfactometry analysis of aroma compounds in a representative organic aroma extract from cured vanilla (Vanilla planifolia G. Jackson) beans. Food Chem 99:728-735

Ramachandra RS, Ravishankar GA (2000) Vanilla flavour: production by conventional and biotechnological routes. J Sci Food Agr 80:298-304

Ranadive AS (1994) Vanilla_cultivation, curing, chemistry, technology and commercial products. In: Charalambrous G (ed) Spices, herbs and edible fungi. Developments in food science, vol 34. Elsevier, Amsterdam, pp 517-577

Röling WFM, Josef K, Martin B, Anton A, Stam H, van Verseveld HW (2001) Microorganisms with a taste for vanilla: microbial ecology of traditional Indonesian vanilla curing. Appl Environ Microb 67:1995-2003 
Sasaki D, Sasaki K, Tsuge Y, Kondo A (2016) Comparative metabolic state of microflora on the surface of the anode electrode in a microbial fuel cell operated at different $\mathrm{pH}$ conditions. AMB Expr 6:125-133

Sharp MD, Kocaoglu-Vurma NA, Langford V, Rodriguez-Saona LE, Harper JW (2012) Rapid discrimination and characterization of vanilla bean extracts by attenuated total reflection infrared spectroscopy and selected ion flow tube mass spectrometry. J Food Sci 77:284-292

Sreedhar RV, Roohie K, Maya P, Venkatachalam L, Bhagyalakshmi N (2009) Biotic elicitors enhance flavor compounds during accelerated curing of vanilla beans. Food Chem 112:461-468

Uzio GLR, Derbesy M (1989) Synthesis, identification and determination of glucosides present in green vanilla beans (Vanilla fragrans Andrews). Flavour Frag J 4:163-167

Walton NJ, Mayer MJ, Narbad A (2003) Vanillin. Phytochemistry 63:505-515
Westcott RJ, Cheetham PSJ, Arraclough AJB (1994) Use of organized viable vanilla plant aerial roots of the production of natural vanillin. Phytochem 35:135-138

Xu F, Zou L, Ong CN (2010) Experiment-originated variations, and multi-peak and multi-origination phenomena in derivatization based GC-MS metabolomics. Trac-Trend Anal Chem 29:269-280

Zenk MH (1965) Biosynthese von vanillin in Vanilla planifolia Andr. Z Pflanzenphysiol 53:404

Zhang SY, Christoph M (2012) Comparative analysis of volatiles in traditionally cured Bourbon and Ugandan vanilla bean (Vanilla planifolia) extracts. J Agr Food Chem 60:10433-10444

Zhao LQ, Sun ZH, Zheng PZ, Lei L (2005) Biotransformation of isoeugenol to vanillin by a novel strain of Bacillus fusiformis. Biotechnol Lett 27:1505-1509

\section{Submit your manuscript to a SpringerOpen ${ }^{\circ}$ journal and benefit from:}

- Convenient online submission

- Rigorous peer review

- Immediate publication on acceptance

- Open access: articles freely available online

- High visibility within the field

- Retaining the copyright to your article 